\title{
Hypoglycaemia: Possible mediation by chromium salt medication
}

\author{
Shabana Valiji Bharmal ${ }^{1}$, Veronica Moyes, Sahid Ahmed ${ }^{2}$, Ashley Grossman ${ }^{1}$ \\ Department of Endocrinology, St. Bartholomew's Hospital, London, ${ }^{2}$ Department of Endocrinology, Great Western \\ Hospital, Swindon, UK
}

\section{INTRODUCTION}

Drugs are the commonest cause of hypoglycaemia in patients without diabetes mellitus. ${ }^{1}$ However, in spite of this, a recent review highlighted the poor quality of evidence available supporting the association between numerous drugs and hypoglycaemia. ${ }^{2}$ In the context of the increasing use of nutritional supplements, appreciation of their potential side effects is ever more important. We report an unusual case of hypoglycaemia which occurred during self-administration of chromium salt supplements, and which has not recurred following supplements discontinuation.

\section{CASE REPORT}

A previously well 53-year old woman presented to the emergency department with acute onset of confusion and drowsiness and a capillary blood glucose of $0.7 \mathrm{mmol} / \mathrm{L}$. There was no relevant past medical or drug history and no excessive alcohol intake. A 72-

Key words: Chromium, Food supplements, Hypoglycaemia

Address for correspondence:

Prof. A.B. Grossman, Department of Endocrinology, St Bartholomew's Hospital, London, EC1A, 7BE, UK Tel.: +44-20 76018343, Fax: +44-20 76018505, e-mail: A.B.Grossman@qmul.ac.uk

Received 26-09-09, Revised 10-12-09, Accepted 20-01-10 hour fast was locally initiated but terminated after 42 hours, at which time a blood glucose of $1.9 \mathrm{mmol} / \mathrm{L}$, plasma insulin $1 \mathrm{mIu} / \mathrm{L}$ ('normal' range $3-20 \mathrm{mIu} / \mathrm{L}$ ) and plasma C-peptide of $126 \mathrm{pmol} / \mathrm{L}$ (normal range $<200 \mathrm{pmol} / \mathrm{L}$ ) was observed. A urinary sulphonylurea screen was negative. The serum IGF-I, calcium and cortisol levels were normal. No pancreatic lesions were identified on cross-sectional abdominal imaging with CT scanning.

In view of the clear-cut hypoglycaemia (i.e. symptoms of neuroglycopaenia with a blood glucose $<2.2 \mathrm{mmol} / \mathrm{L}$ ) and absence of inappropriate insulin or C-peptide excess, the patient was referred for further investigation. A mixed meal test performed 5 months after the initial presentation demonstrated no reactive hypoglycaemia (Table 1). A 72-hour fast, followed by brief intense exercise, was completed without hypoglycaemia; the lowest plasma glucose was $2.7 \mathrm{mmol} / \mathrm{L}$ after exercise with a plasma insulin level of $2.2 \mathrm{mIu} / \mathrm{L}$ and a C-peptide of $<94 \mathrm{pmol} / \mathrm{L}$.

In the light of these findings, the patient's history was reviewed in more detail. She then admitted that for 18 months prior to the initial presentation she had been taking "adrenal supplements" obtained from a health food store, but she had not considered this information relevant; via these supplements, the patient was receiving $200 \mathrm{mcg}$ chromium picolinate daily, whereas the recommended adequate intake for chromium for adult women is $20 \mathrm{mcg} / \mathrm{d} .{ }^{3}$ These supplements were discontinued after her initial presentation and subsequent hospital admission. A 00:90 cortisol 
Table 1. Results of mixed meal test

\begin{tabular}{lcc}
\hline Time (minutes) & Glucose $(\mathbf{m m o l} / \mathbf{L})$ & Insulin $(\mathbf{m I u} / \mathbf{L})$ \\
\hline-30 & 5.1 & 4.5 \\
0 (mixed meal) & 5.1 & 4.3 \\
30 & 4.2 & 50 \\
60 & 4.0 & 35 \\
90 & 3.3 & 24 \\
120 & 3.3 & 15 \\
150 & 2.9 & 14 \\
180 & 3.3 & 9.6 \\
210 & 3.6 & 6.5 \\
240 & 4.2 & 5.9 \\
270 & 4.4 & 5.9 \\
300 & 4.4 & 3.8 \\
\hline
\end{tabular}

value of $750 \mathrm{nmol} / 1$ excluded cortisol deficiency as a cause of her hypoglycaemia. She has had no further hypoglycaemic episodes and remained well 6 months after her initial hospital admission.

\section{DISCUSSION}

The patient in question demonstrated unequivocal hypoglycaemic episodes with suppressed insulin and C-peptide levels, which could not be replicated on her second hospital admission. The only difference between the two admissions was the cessation of her chromium supplements. There was never any evidence in favour of a mass lesion secreting other hypoglycaemic substances such as IGF-II. We therefore tentatively suggest that the chromium supplements were instrumental in the hypoglycaemic episodes.

Chromium picolinate has been demonstrated to enhance insulin sensitivity, this effect having been utilised in patients with type 2 diabetes mellitus to improve glycaemic control. A randomised study of 25 patients with type 2 diabetes treated for 24 weeks with glipizide plus $1000 \mathrm{mcg}$ chromium picolinate daily, versus glipizide plus placebo, demonstrated improved glycaemic control and increased insulin sensitivity in those receiving chromium picolinate. ${ }^{4}$ Further reports suggest chromium as the cause of spontaneous hypoglycaemia: Whipple's triad (symptoms consistent with hypoglycaemia, a low plasma glucose concentration and resolution of hypoglycaemic symptoms by glucose administration) was documented in a patient receiving $200 \mathrm{mcg} / \mathrm{day}$ chromium in addition to their usual insulin dose for the management of type 1 diabetes mellitus. ${ }^{5}$ Following this episode, the patient was prescribed $1000 \mathrm{mcg} /$ day chromium without insulin and had another similar episode 6 months later. However, there have been no case reports of spontaneous hypoglycaemia in non-diabetic patients.

A proposed molecular mechanism consistent with in vivo observations is that chromium mobilises the glucose transporter to the inner cell surface membrane. ${ }^{6}$ With insulin, these transporters are then physically incorporated into the plasma membrane, leading to an overall reduction in plasma glucose concentration; in the absence of insulin, these chromium-mobilised glucose transporters are said not to be active.

In our patient one might have expected that any change in insulin sensitivity would have been compensated for by changes in insulin secretion, thus avoiding hypoglycaemia. We have no explanation as to why chromium salts may have caused true hypoglycaemia in this case and admit that the evidence linking the chromium administration is purely circumstantial. However, there is no doubt that the patient became hypoglycaemic on fasting with appropriately suppressed levels of insulin, and that after cessation of the chromium supplements no hypoglycaemia could be induced. The patient refused the possibility of rechallenging with chromium supplements.

\section{CONCLUSIONS}

Increasing numbers of patients are now taking nutritional supplements and over-the-counter remedies which are frequently not disclosed during routine assessment. Our case highlights the need for a detailed and comprehensive drug history, including nutritional supplements, in the assessment of patients with unexplained spontaneous hypoglycaemia.

The long half-life and consequently unpredictable effects of chromium, as illustrated in our case, emphasise the need for further research into safe daily requirements of chromium in the non-deficient non-diabetic population and its long-term toxicity effects. 


\section{REFERENCES}

1. Marks V, Teale JD,1999 Drug-induced hypoglycemia. Endocrinol Metab Clin North Am 28: 555-577.

2. Murad MH, Coto-Yglesias F, Wang AT, et al, 2009 Drug-Induced Hypoglycemia: A Systematic Review. J Clinl Endocrinol Metab 94: 741-745.

3. Food and Nutrition Board: Dietary Reference Intakes for Vitamin A, Vitamin K, Arsenic, Boron, Chromium, Copper, Iodine, Iron, Manganese, Molybdenum, Nickel, Silicon, Vanadium, and Zinc 2001, National Academy Press, Washington; DC.
4. Martin J, Wang ZQ, Zhang XH, et al, 2006 Chromium picolinate supplementation attenuates body weight gain and increases insulin sensitivity in subjects with type 2 diabetes. Diabetes Care 29: 1826-1832.

5. Bunner S, McGinnis R, 1998 Chromium-Induced Hypoglycemia. Psychosomatics 39: 298-299.

6. Chen G, Liu P, Pattar GR, et al, 2006 Chromium Activates Glucose Transporter 4 Trafficking and Enhances Insulin-Stimulated Glucose Transport in 3T3-L1 Adipocytes via a Cholesterol-Dependent Mechanism. Mol Endocrinol 20: 857-870. 